\title{
A Systematic Derivation of the Riemannian
}

\section{Barrett-Crane Intertwiner.}

\author{
Suresh. K. Maran
}

October 28, 2018

\begin{abstract}
The Barrett-Crane intertwiner for the Riemannian general relativity is systematically derived by solving the quantum Barrett-Crane constraints corresponding to a tetrahedron (except for the non-degeneracy condition). It was shown by Reisenberger that the Barrett-Crane intertwiner is the unique solution. The systematic derivation can be considered as an alternative proof of the uniqueness. The new element in the derivation is the rigorous imposition of the cross-simplicity constraint.
\end{abstract}

\section{Introduction}

A quantization of a four-simplex for the Riemannian general relativity was proposed by Barrett and Crane [1. It was built on the idea of the Barrett-Crane intertwiner. It was shown by Reisenberger [2] that the Barrett-Crane intertwiner is the unique solution to the Barrett-Crane constraints corresponding a tetrahedron (except for the non-degeneracy condition). Here I would I like to present an alternative proof of uniqueness by systematically deriving the Barrett-Crane intertwiner by imposing the Barrett-Crane constraints. 


\section{Review}

The bivectors $B_{i}$ associated with the ten triangles of a four-simplex in a flat Riemannian space satisfy the following properties called the Barrett-Crane constraints [1:

1. The bivector changes sign if the orientation of the triangle is changed.

2. Each bivector is simple.

3. If two triangles share a common edge, then the sum of the bivectors is also simple.

4. The sum of the bivectors corresponding to the edges of any tetrahedron is zero. This sum is calculated taking into account the orientations of the bivectors with respect to the tetrahedron.

5. The six bivectors of a four-simplex sharing the same vertex are linearly independent.

6. The volume of a tetrahedron calculated from the bivectors is real and non-zero.

The items two and three can be summarized as follows:

$$
B_{i} \wedge B_{j}=0 \forall i, j
$$

where $A \wedge B=\varepsilon_{I J K L} A^{I J} B^{K L}$ and the $i, j$ represents the triangles of a tetrahedron. If $i=j$, it is referred to as the simplicity constraint. If $i \neq j$ it is referred as the cross-simplicity constraints.

Barrett and Crane have shown [1] that these constraints are sufficient to restrict a general set of ten bivectors $E_{b}$ so that they correspond to the triangles 
of a geometric four-simplex up to translations and rotations in a four dimensional flat Riemannian space.

A quantum four-simplex for Riemannian general relativity is defined by quantizing the Barrett-Crane constraints [1]. The bivectors $B_{i}$ are promoted to the Lie operators $\hat{B}_{i}$ on the representation space of the relevant group and the Barrett-Crane constraints are imposed at the quantum level. The last two constraints are inequalities and they are difficult to impose. For these reasons here after I would like to refer to a state sum model that satisfies only the first four constraints as an essential Barrett-Crane model, While a state sum model that satisfies all the six constraints as a rigorous Barrett-Crane model. The Barrett-Crane intertwiner corresponds to essential Barrett-Crane model only. We will do a systematic derivation of the essential Barrett-Crane model here.

\subsection{The Simplicity Constraint}

Our treatment of the simplicity constraints is basically a review of work done

before [1], 3. The group $S O(4, R)$ is isomorphic to $\frac{S U(2) \times S U(2)}{Z_{2}}$. An element $B$ of the Lie algebra of $S O(4)$ can be split into the left and the right handed $S U(2)$ components,

$$
B=B_{L}+B_{R}
$$

There are two Casimir operators for $S O(4)$ which are

$$
\begin{aligned}
& \varepsilon_{I J K L} B^{I J} B^{K L} \text { and } \\
& \delta_{I K} \delta_{J L} B^{I J} B^{K L},
\end{aligned}
$$

where $\eta_{I K}$ is the flat Euclidean metric. In terms of the left and right handed split I can expand the Casimir operators as

$$
\varepsilon_{I J K L} B^{I J} B^{K L}=B_{L} \cdot B_{L}-B_{R} \cdot B_{R} \text { and }
$$




$$
\delta_{I K} \delta_{J L} B^{I J} B^{K L}=B_{L} \cdot B_{L}+B_{R} \cdot B_{R}
$$

where the dot products are the trace in the $S U(2)$ Lie algebra coordinates.

The bivectors are to be quantized by promoting the Lie algebra vectors to Lie operators on the unitary representation space of $S O(4) \cong \frac{S U(2) \times S U(2)}{Z_{2}}$. The relevant unitary representations of $S O(4)$ are labeled by a pair $\left(J_{L}, J_{R}\right)$ of unitary representations of $S U(2)$. The elements of the representation space $D_{J_{L}} \otimes D_{J_{R}}$ are the eigen states of the Casimirs and on them the operators reduce to the following:

$$
\begin{gathered}
\varepsilon_{I J K L} \hat{B}^{I J} \hat{B}^{K L}=\frac{J_{L}\left(J_{L}+1\right)-J_{R}\left(J_{R}+1\right)}{2} \hat{I} \text { and } \\
\delta_{I K} \delta_{J L} \hat{B}^{I J} \hat{B}^{K L}=\frac{J_{L}\left(J_{L}+1\right)+J_{R}\left(J_{R}+1\right)}{2} \hat{I} .
\end{gathered}
$$

The equation (2) implies that on $D_{J_{L}} \otimes D_{J_{R}}$ the simplicity constraint $B \wedge B=0$ is equivalent to the condition $J_{L}=J_{R}$. I would like to find a representation space on which the representations of $S O(4)$ are restricted precisely by $J_{L}=$ $J_{R}$.

In Ref: [3] it has been shown for $S O(N, R)$ that the simplicity constraint reduces the Hilbert space associated to a triangle to that of the $L^{2}$ functions on $S^{N-1}$. Consider a square integrable function $f(x)$ on the sphere $S^{3}$ defined by

$$
x \cdot x=1, \forall x \in \boldsymbol{R}^{4} .
$$

It can be Fourier expanded in the representation matrices of $S U(2)$ using the isomorphism $S^{3} \cong S U(2)$,

$$
f(x)=\sum_{J} d_{J} \operatorname{Tr}\left(F_{J} T_{J}\left(\mathfrak{g}(x)^{-1}\right),\right.
$$

where $\mathfrak{g}: S^{3} \longrightarrow S U(2)$ is an isomorphism from $S^{3}$ to $S U(2), F_{m_{2} J}^{m_{1}}$ the Fourier coefficients, $T_{m_{2} J}^{m_{1}}(g)$ are the matrix elements of spin $J$ representation of an 
element $g \in S U(2)$ and $d_{J}$ the dimension of the $J$ representation. The group action of $g=\left(g_{L}, g_{R}\right) \in S O(4)$ on $x \in S^{3}$ is given by

$$
\mathfrak{g}(g x)=g_{L}^{-1} \mathfrak{g}(x) g_{R}
$$

Using equation (4) I can consider the $T_{J}(\mathfrak{g}(x))\left(m_{1}, m_{2}\right)$ as the basis functions of $L^{2}$ functions on $S^{3}$. The matrix elements of the action of $g$ on $S^{3}$ is given by

$$
\int \bar{T}_{\dot{m}_{2} \dot{J}}^{\dot{m}_{1}}(\mathfrak{g}(x)) T_{m_{2} J}^{m_{1}}(\mathfrak{g}(g x)) d x=\frac{1}{d_{J}} \bar{T}_{m_{1} \dot{J}}^{\dot{m}_{1}}\left(g_{L}\right) T_{m_{2} J}^{\dot{m}_{2}}\left(g_{R}\right) \delta(\dot{J}-J) .
$$

I see that the representation matrices are precisely those of $S O(4)$ only restricted by the constraint $J_{L}=J_{R}$. Since we have all the simple representations included in the representation, the simplicity constraint effectively reduces the Hilbert space $H$ to the space of $L^{2}$ functions on $S^{3}$.

\subsection{The Cross-Simplicity Constraints}

Next let me quantize the cross-simplicity constraint part of the Barrett-Crane constraint. Consider the quantum state space associated with a pair of triangles 1 and 2 of a tetrahedron. A general quantum state that just satisfies the simplicity constraints $B_{1} \wedge B_{1}=0$ and $B_{2} \wedge B_{2}=0$ is of the form $f\left(x_{1}, x_{2}\right)$ $\in L^{2}\left(S^{3} \times S^{3}\right), x_{1}, x_{2} \in S^{3}$.

On the elements of $L^{2}\left(S^{3} \times S^{3}\right)$ the action $B_{1} \wedge B_{2}$ is equivalent to the action of $\left(B_{1}+B_{2}\right) \wedge\left(B_{1}+B_{2}\right)^{1}$. This implies that the cross-simplicity constraint $B_{1} \wedge B_{2}=0$ requires the simultaneous rotation of $x_{1}, x_{2}$ involve only the $J_{L}$

${ }^{1}$ Please notice that

$$
\left(\hat{B}_{1}+\hat{B}_{2}\right) \wedge\left(\hat{B}_{1}+\hat{B}_{2}\right)=\hat{B}_{1} \wedge \hat{B}_{1}+\hat{B}_{2} \wedge \hat{B}_{2}+2 B_{1} \wedge \hat{B}_{2} .
$$

But since $\hat{B}_{1} \wedge \hat{B}_{1}=\hat{B}_{2} \wedge \hat{B}_{2}=0$ on $f\left(x_{1}, x_{2}\right)$ we have

$$
\left(\hat{B}_{1}+\hat{B}_{2}\right) \wedge\left(\hat{B}_{1}+\hat{B}_{2}\right) f\left(x_{1}, x_{2}\right)=\hat{B}_{1} \wedge \hat{B}_{2} f\left(x_{1}, x_{2}\right) .
$$


$=J_{R}$ representations. The simultaneous action of $g=\left(g_{L}, g_{R}\right)$ on the arguments of $f\left(x_{1}, x_{2}\right)$ is

$$
g f\left(x_{1}, x_{2}\right)=f\left(g_{L}^{-1} x_{1} g_{R}, g_{L}^{-1} x_{2} g_{R}\right) .
$$

The harmonic expansion of $f\left(x_{1}, x_{2}\right)$ in terms of the basis function $T_{J}(\mathfrak{g}(x))\left(m_{1}, m_{2}\right)$ is

$$
f\left(x_{1}, x_{2}\right)=\sum_{J} F_{m_{1} m_{2} J_{1} J_{2}}^{\dot{m}_{1} \dot{m}_{2}} T_{\dot{m}_{1} J_{1}}^{m_{1}}\left(\mathfrak{g}\left(x_{1}\right)\right) T_{\dot{m}_{2} J_{2}}^{m_{2}}\left(\mathfrak{g}\left(x_{2}\right)\right) .
$$

The rest of the calculations can be understood graphically. The last equation can be graphically written as follows:

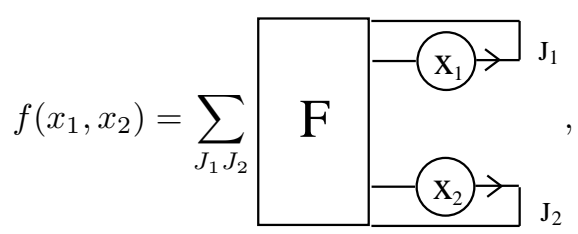

where the box $F$ represents the Fourier coefficient $F_{m_{1} m_{2} J_{1} J_{2}}^{\dot{m}_{1} \dot{m}_{2}}$. The action of $g \in S O(4)$ on $f$ is

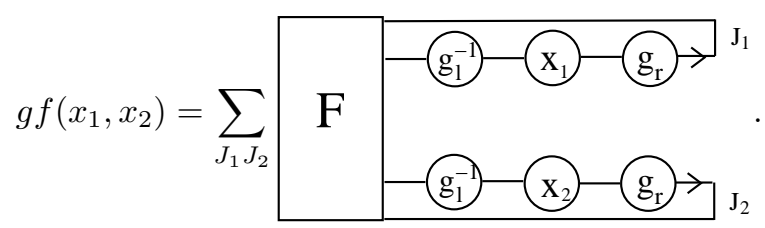

Now for any $h \in S U(2)$,

$$
T_{a_{1} J_{1}}^{b_{1}}(h) T_{a_{2} J_{1}}^{b_{2}}(h)=\sum_{J_{3}} C_{J_{1} J_{2} b_{3}}^{b_{1} b_{2} J_{3}} \bar{C}_{a_{1} a_{2} J_{3}}^{J_{1} J_{2} a_{3}} T_{a_{3} J_{3}}^{b_{3}}(h),
$$

where $C$ 's are the Clebsch-Gordan coefficients of $S U(2)$ 4. I have assumed all the repeated indices are either integrated or summed over for the previous and the next two equations. Using this I can rewrite the $g_{L}$ and $g_{R}$ parts of the result (7) as follows:

$$
T_{a_{1} J_{1}}^{m_{1}}\left(g_{L}^{-1}\right) T_{a_{2} J_{2}}^{m_{2}}\left(g_{L}^{-1}\right)=\sum_{J_{L}} C_{J_{1} J_{2} m_{3}}^{m_{1} m_{2} J_{L}} \bar{C}_{a_{1} a_{2} J_{L}}^{J_{1} J_{2} a_{3}} T_{a_{3} J_{L}}^{m_{3}}\left(g_{L}^{-1}\right)
$$


and

$$
T_{\dot{m}_{1} J_{1}}^{b_{1}}\left(g_{R}\right) T_{\dot{m}_{2} J_{2}}^{b_{2}}\left(g_{R}\right)=\sum_{J_{L}} C_{J_{1} J_{2} b_{3}}^{b_{1} b_{2} J_{R}} \bar{C}_{\dot{m}_{1} \dot{m}_{2} J_{R}}^{J_{1} J_{2} \dot{m}_{3}} T_{\dot{m}_{3} J_{R}}^{b_{3}}\left(g_{R}\right)
$$

Now we have

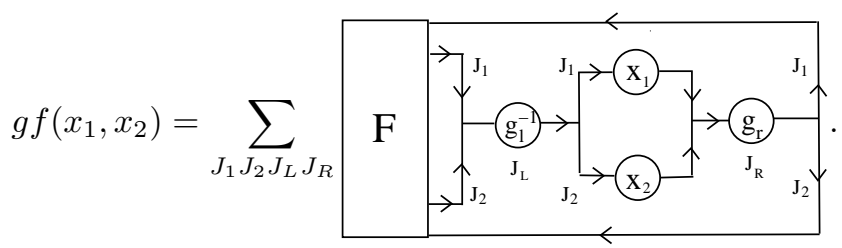

To satisfy the cross-simplicity constraint the expansion of $g f\left(x_{1}, x_{2}\right)$ must have contribution only from the terms with $J_{L}=J_{R}$. Let me remove all the terms which do not satisfy $J_{L}=J_{R}$. Also let me set $g=I$. Now we can deduce that the functions denoted by $\tilde{f}\left(x_{1}, x_{2}\right)$ obtained by reducing $f\left(x_{1}, x_{2}\right)$ using the cross-simplicity constraints must have the expansion,

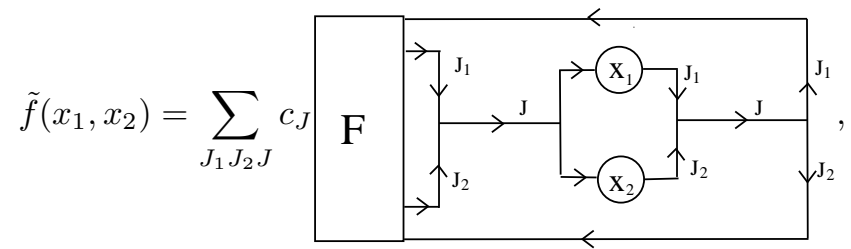

where the coefficients $c_{J}$ introduced depends on the precise definition of the cross-simplicity projector. But as we will see, the final answer does not depend on the $c_{J}$ 's. Now the Clebsch-Gordan coefficient terms in the expansion can be re-expressed using the following equation :

$$
C_{J_{1} J_{2} m_{3}}^{m_{1} m_{2} J} \bar{C}_{\dot{m}_{1} \dot{m}_{2} J}^{J_{1} J_{2} \dot{m}_{3}}=\frac{1}{d_{J}} \int_{S U(2)} T_{\dot{m}_{1} J_{1}}^{m_{1}}(h) T_{\dot{m}_{2} J_{2}}^{m_{2}}(h) \bar{T}_{m_{3} J}^{\dot{m}_{3}}(h) d h,
$$

where $h, \tilde{h} \in S U(2)$ and $d h$ the bi-invariant measure on $S U(2)$. Using this in the two middle Clebsch-Gordan coefficients of $\tilde{f}\left(x_{1}, x_{2}\right)$ we get

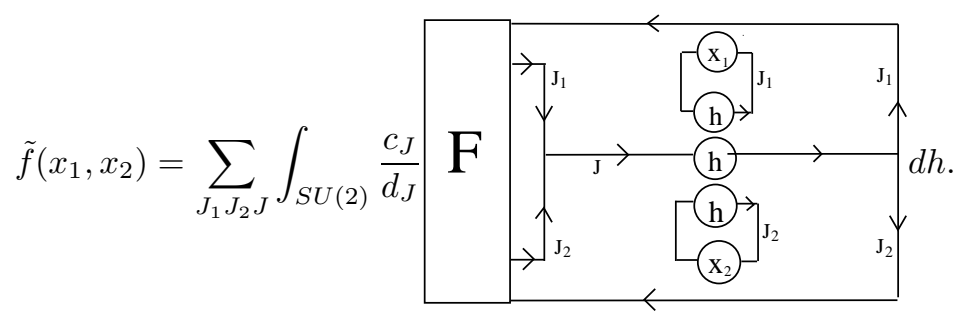


This result can be rewritten for clarity as

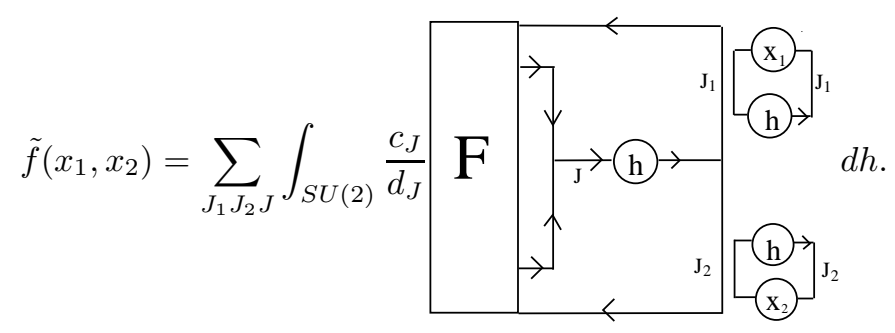

Once again applying equation (11) to the remaining two Clebsch-Gordan coefficients we get,

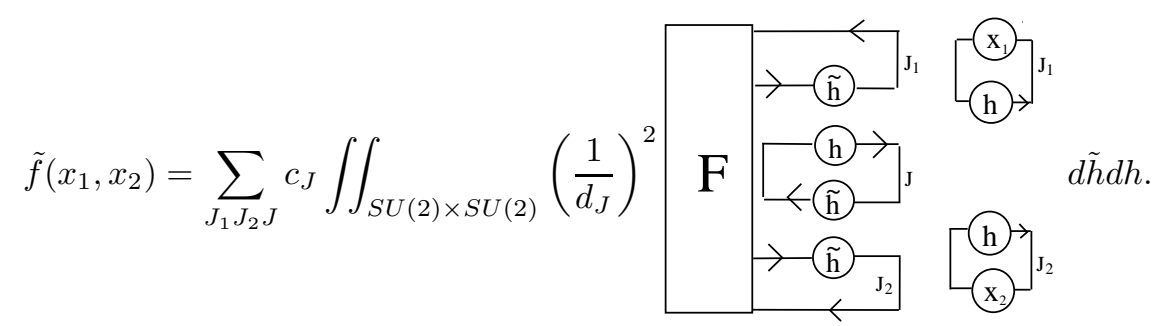

By rewriting the above expression, I deduce that a general function $\tilde{f}\left(x_{1}, x_{2}\right)$ that satisfies the cross-simplicity constraint must be of the form,

$$
\begin{aligned}
\tilde{f}\left(x_{1}, x_{2}\right) & =\sum_{J_{1} J_{2}} \int_{S U(2)} F_{J_{1} J_{2}}(h) \\
& =\sum_{J_{1} J_{2}} \int_{S U(2)} F_{J_{1} J_{2}}(h) \operatorname{tr}\left(T _ { J _ { 1 } } ( \mathfrak { g } ( x _ { 1 } ) h ) \operatorname { t r } \left(T_{J_{2}}\left(\mathfrak{g}\left(x_{2}\right) h\right) d h,\right.\right.
\end{aligned}
$$

where $F_{J_{1} J_{2}}(h)$ is arbitrary.

If $\Psi\left(x_{1}, x_{2}, x_{3}, x_{4}\right)$ is the quantum state of a tetrahedron that satisfies all of the simplicity constraints and the cross-simplicity constraints, it must be of the form,

$$
\begin{aligned}
& \Psi\left(x_{1}, x_{2}, x_{3}, x_{4}\right) \\
& =\sum_{J_{1} J_{2} J_{3} J_{4}} F_{J_{1} J_{2} J_{3} J_{4}}(h) \operatorname{tr}\left(T _ { J _ { 1 } } ( \mathfrak { g } ( x _ { 1 } ) h ) \operatorname { t r } \left(T_{J_{2}}\left(\mathfrak{g}\left(x_{2}\right) h\right)\right.\right. \\
& \operatorname{tr}\left(T _ { J _ { 3 } } ( \mathfrak { g } ( x _ { 3 } ) h ) \operatorname { t r } \left(T_{J_{4}}\left(\mathfrak{g}\left(x_{4}\right) h\right) d h .\right.\right.
\end{aligned}
$$


This general form is deduced by requiring that for every pair of variables with the other two fixed, the function must be the form of the right hand side of equation (12).

\subsection{The $S O(4)$ Barrett-Crane Intertwiner}

Now the quantization of the fourth Barrett-Crane constraint demands that $\Psi$ is invariant under the simultaneous rotation of its variables. This is achieved if $F_{J_{1} J_{2} J_{3} J_{4}}(h)$ is a constant function of $h$. Therefore the quantum state of a tetrahedron is spanned by

$$
\Psi_{J_{1} J_{2} J_{3} J_{4}}\left(x_{1}, x_{2}, x_{3}, x_{4}\right)=\int_{n \in S^{3}} \prod_{i} T_{J_{i}}\left(\mathfrak{g}\left(x_{i}\right) \mathfrak{g}(n)\right) d n,
$$

where the measure $d n$ on $S^{3}$ is derived from the bi-invariant measure on $S U(2)$.

The quantum state can be diagrammatically represented as follows:

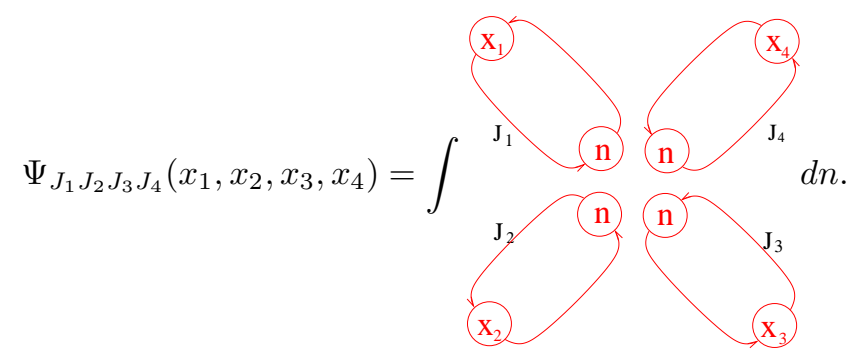

A unitary representation $T_{J}$ of $S U(2)$ can be considered as an element of $D_{J} \otimes D_{J}^{*}$

where $D_{J}^{*}$ is the dual representation of $D_{J}$. So using this the Barrett-Crane intertwiner can be written as an element $\left|\Psi_{J_{1} J_{2} J_{3} J_{4}}\right\rangle \in \bigotimes_{i} D_{J_{i}} \otimes D_{J_{i}}^{*}$ as follows:

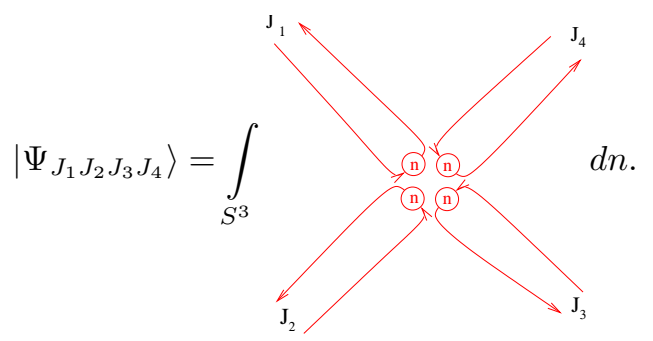

Since $S U(2) \approx S^{3}$, using the following graphical identity: 


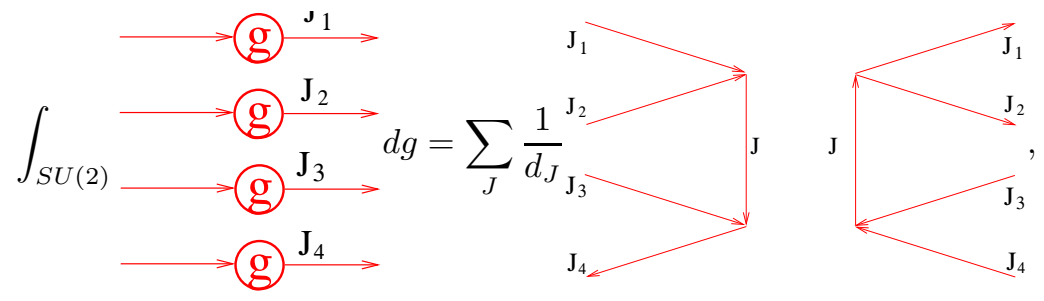

the Barrett-Crane solution can be rewritten as

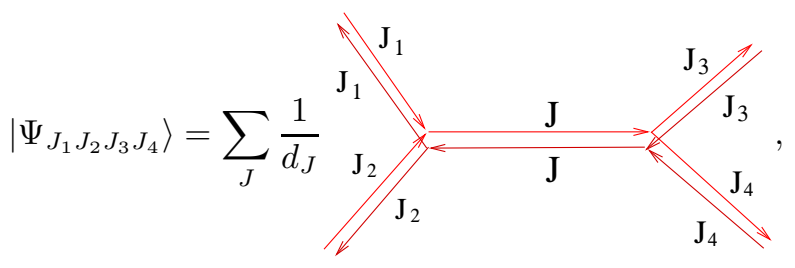

which emerges as an intertwiner in the familiar form in which Barrett and Crane proposed it for the Riemannian general relativity.

\section{References}

[1] J. W. Barrett and L. Crane, Relativistic Spin Networks and Quantum general relativity. J.Math.Phys., 39:3296-3302, 1998.

[2] M. P. Reisenberger, On Relativistic Spin Network Vertices, J.Math.Phys., 40:2046-2054, 1999.

[3] L. Freidel, K. Krasnov and R. Puzio, BF Description of HigherDimensional Gravity Theories, Adv. Theor. Math. Phys. 3 (1999) 1289-1324, arXiv:hep-th/9901069

[4] D. A. Varshalovich, A. N. Moskalev and V. K. Khersonskii, Quantum Theory of Angular Momentum, World Scientific, 1988. 